\title{
Role of higher twist effects in diffractive DIS and determination of diffractive parton distribution functions
}

\author{
Atefeh Maktoubian, ${ }^{1, *}$ Hossein Mehraban, ${ }^{1, \dagger}$ Hamzeh Khanpour $\oplus^{2,3, \$}$ and Muhammad Goharipour $\oplus^{3, \S}$ \\ ${ }^{1}$ Faculty of Physics, Semnan University, P.O.Box 35131-19111, Semnan, Iran \\ ${ }^{2}$ Department of Physics, University of Science and Technology of Mazandaran, \\ P.O.Box 48518-78195, Behshahr, Iran \\ ${ }^{3}$ School of Particles and Accelerators, Institute for Research in Fundamental Sciences (IPM), \\ P.O.Box 19395-5531, Tehran, Iran
}

(Received 1 August 2019; published 17 September 2019)

\begin{abstract}
The current analysis aims to present the results of a QCD analysis of diffractive parton distribution functions (PDFs) at next-to-leading-order accuracy in perturbative QCD. In this new determination of diffractive PDFs, we use all available and up-to-date diffractive deep-inelastic scattering (DIS) datasets from $\mathrm{H} 1$ and ZEUS collaborations at HERA, including the most recent H1/ZEUS combined measurements. In this analysis, we consider the heavy quark contributions to the diffractive DIS in the so-called framework of the FONLL general mass variable flavor number scheme. The uncertainties on the diffractive PDFs are calculated using the standard "Hessian error propagation," which served to provide a more realistic estimate of the uncertainties. This analysis is enriched, for the first time, by including the nonperturbative higher twist (HT) effects in the calculation of diffractive DIS cross sections, which are particularly important at large- $x$ and low $Q^{2}$ regions. Then, the stability and reliability of the extracted diffractive PDFs are investigated upon the inclusion of HT effects. We discuss the novel aspects of the approach used in this QCD fit, namely, optimized and flexible parametrizations of diffractive PDFs, the inclusion of HT effects, and considering the recent H1/ZEUS combined dataset. Finally, we present the extracted diffractive PDFs with and without the presence of HT effects and discuss the fit quality and the stability upon variations of the kinematic cuts and the fitted datasets. We show that the inclusion of HT effects in diffractive DIS can improve the description of the data, which leads, in general, to a very good agreement between data and theory predictions.
\end{abstract}

DOI: 10.1103/PhysRevD.100.054020

\section{INTRODUCTION}

Over the past few decades, it has been known that the parton distribution functions (PDFs) of nucleons are an essential ingredient for the interpretation and QCD phenomenology of hadron structure in high-energy experiments such as the deep-inelastic lepton-nucleon scattering $(\ell p$ DIS $)$ and hadron-hadron collisions. Despite the active experimental and theoretical investigations, the determination of PDFs along with their uncertainties through a global QCD analysis is still an important topic in high-energy physics. In recent years, there has been an increasing amount of literature on

\footnotetext{
*Atefeh.Maktoubian@semnan.ac.ir

${ }^{\dagger}$ Hmehraban@semnan.ac.ir

${ }^{*}$ Hamzeh.Khanpour@cern.ch

${ }^{\S}$ Muhammad.Goharipour@ipm.ir
}

Published by the American Physical Society under the terms of the Creative Commons Attribution 4.0 International license. Further distribution of this work must maintain attribution to the author(s) and the published article's title, journal citation, and DOI. Funded by SCOAP ${ }^{3}$. this topic. For more detailed discussions, we refer the reader to Refs. [1-6] for introductory texts on the fundamentals of QCD factorization, global QCD PDFs analyses, and phenomenological applications of PDFs in the LHC era.

Among the high-energy experiments, the most interesting physics results mainly came from the $\mathrm{H} 1$ and ZEUS experiments at HERA-I and HERA-II, which have provided an impressive wealth of information about the proton structure. In addition, among the high-energy processes of interest, diffractive DIS, which contributes a fraction of order $8 \%-10 \%$ to the total DIS cross section, also aims to discover the underlying structure of hadrons through diffractive processes [7-9]. The method of global QCD analysis for diffractive PDFs is the same as the ordinary PDFs. It is also based on the QCD factorization of physical observables. According to the factorization theorem for diffractive DIS in perturbative QCD [10-12], the hard scattering cross sections can be expressed as a convolution between the hard partonic cross sections, which can be calculated in perturbative QCD, and the nonperturbative diffractive PDFs. The latter need to be extracted from QCD 
analyses of a variety of available hard scattering diffractive DIS experimental datasets, though the inclusion of other diffractive data from collider experiments can improve their uncertainties to a significant extent.

Recent progresses in global QCD analysis of diffractive PDFs have used all available high-precision measurements from the $\mathrm{H} 1$ and ZEUS collaborations at HERA, which have led to a precise determination of diffractive PDFs. In recent years, a considerable amount of literature has been published on diffractive PDFs analysis with uncertainties. These include the most recent analysis by GKG18-DPDF [13], which has been done in the framework of xFitter [14] considering the most recent H1/ZEUS combined dataset [15]; H1-2006-DPDF [16]; and ZEUS-2010-DPDF [17]. All these analyses were performed at next-to-leading-order (NLO) accuracy in QCD. More recently, HK19-DPDF [18] has reported sets of diffractive PDFs at NLO and, for the first time, at next-to-next-to-leading-order accuracy in perturbative QCD by analyzing all available and up-todate datasets for diffractive DIS including the H1/ZEUS combined measurements on hard scattering diffractive cross sections [15].

The current analysis aims to clarify whether the inclusion of nonperturbative higher twist (HT) effects can affect the QCD analysis of diffractive DIS data and hence can fill a gap in the QCD analysis of diffractive PDFs in literature. To this end, we analyzed the diffractive DIS datasets through QCD analyses with or without the inclusion of HT terms. We observed that 1) the HT effects may have significant contributions in diffractive DIS cross sections, 2) considering HT effects in the QCD analysis of diffractive PDFs could affect the data/theory comparisons and significantly improve the fit quality, and 3) considering HT effects in the QCD analysis of diffractive PDFs could also affect the shape and size of the extracted diffractive PDFs. From the comparisons presented in this study, we see a number of interesting similarities and differences between these two diffractive PDF sets and their uncertainties. We will return to this issue in more details in Sec. IV.

The outline of this article is the following. In Sec. II, we introduce the theoretical framework and kinematical variables used for the definition of diffractive DIS processes, diffractive structure functions, and diffractive reduced cross sections. In this section, we also present our parametrization for the quark and gluon diffractive PDFs, the heavy quark contributions in the general mass variable flavor number scheme (GM-VFNS) [19-21], and finally the formalism of HT effects used in this analysis. Section III includes a comprehensive introduction of the diffractive DIS experimental datasets that are used in our diffractive PDFs analysis. The main results and findings of the present diffractive PDFs analysis are discussed in details in Sec. IV. In the same section, we present the extracted diffractive PDFs and detailed comparisons with other results in literature. Then, we assess the quality of our fit by comparing the resulting diffractive reduced cross sections with the experimental datasets. We also assess the stability of our QCD analysis with respect to the inclusion of nonperturbative HT terms. Lastly, in Sec. V, we summarize and discuss our analysis. This section also includes a brief discussion of the implication of the findings for future research.

\section{PHENOMENOLOGICAL FRAMEWORK}

In this section, we discuss in detail the theoretical framework for the evaluation of diffractive DIS structure functions and reduced cross sections, the quark and gluon diffractive PDFs at the input scale, the heavy quark mass effects, the nonperturbative HT corrections, and finally the software tools used for the numerical calculations of diffractive DIS cross sections.

\section{A. Theory settings}

In this section, we review in detail the theoretical formalism that describes diffractive DIS of charged leptons $\left(\ell^{ \pm}\right)$of proton $(p)$ at HERA. As mentioned in the Introduction, the neutral current diffractive DIS process $e p \rightarrow e p X$, where $X$ represents the hadronic final state, is a DIS process in which a percent of the interacting proton remains intact. It has shown that, in $e p$ collisions at HERA, this hard diffraction contributes a fraction of order $8 \%-10 \%$ to the total DIS cross sections [16]. Such a process can be explained in terms of particle exchanges with the net vacuum quantum numbers, namely, Pomeron and Reggeon, though there are different theoretical approaches to describe this process [22]. Figure 1 shows the schematic parton model diagram of inclusive diffractive DIS $e p \rightarrow e p X$. Four-momenta are indicated in parentheses as well. The variable $\beta$ is the momentum fraction of the struck quark.

Note that the hadronic final state $X$ is separated from the intact proton by a rapidity gap (RG), so most of the momentum is carried by the proton. We start now by briefly reviewing the definition of the diffractive DIS

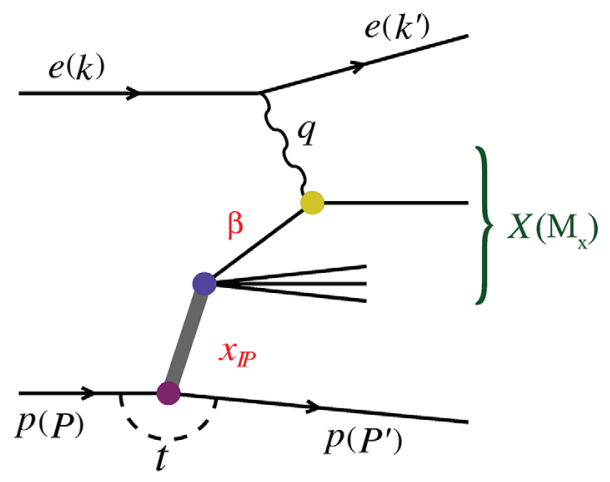

FIG. 1. A schematic parton model diagram of inclusive diffractive DIS $e p \rightarrow e p X$. Four-momenta are indicated in parentheses as well. The variable $\beta$ is the momentum fraction of the struck quark. 
reduced cross sections and of the associated kinematical variables, which are relevant for the description of the diffractive lepton-proton $\left(\ell^{ \pm} p\right)$ scattering. As can be seen from Fig. 1, in addition to the common variables for describing DIS, which are the photon virtuality $Q^{2}=$ $-q^{2}=-\left(k-k^{\prime}\right)^{2}$, the longitudinal momentum fraction $x$ and the inelasticity $y$, one needs also some other essential variables for describing diffractive DIS. These new variables are as follow. The mass of the diffractive final state $M_{X}$ that is usually replaced by the Bjorken variable defined for the diffractive exchange $\beta$. The second variable is the squared 4-momentum transferred at the proton vertex $t$ which indicates the square of the difference between the 4-momentum of the outgoing and incoming protons, $P$ and $P^{\prime}$. The fraction of the momentum of proton carried by the diffractive exchange, which is denoted by $x_{\mathbb{P}}$, is related to $x$ and $\beta$ variables through $x_{\mathbb{P}}=x / \beta$.

The only available diffractive DIS datasets come from HERA $e p$ collider measurements that are provided by the H1 and ZEUS collaborations [15-17,23-31]. These measurements are usually presented in terms of the so-called diffractive reduced cross sections $\sigma_{r}^{D(4)}\left(\beta, Q^{2} ; x_{\mathbb{P}}, t\right)$, which depend on the diffractive structure functions, $F_{2}^{D(4)}$ and $F_{L}^{D(4)}$, according to the following equation:

$$
\begin{aligned}
\sigma_{r}^{D(4)}\left(\beta, Q^{2} ; x_{\mathbb{P}}, t\right)= & F_{2}^{D(4)}\left(\beta, Q^{2} ; x_{\mathbb{P}}, t\right) \\
& -\frac{y^{2}}{1+(1-y)^{2}} F_{L}^{D(4)}\left(\beta, Q^{2} ; x_{\mathbb{P}}, t\right) .
\end{aligned}
$$

In the experimental point of view, the longitudinal structure function, $F_{L}^{D(4)}$, is small enough and can be neglected in some specific limits where $y$ is not to close to unity. As can be easily checked, if one neglects $F_{L}^{D^{(4)}}$, the diffractive reduced cross section $\sigma_{r}^{D(4)}$ will be approximately equal to $F_{2}^{D(4)}$. It is worth noting here that the diffractive DIS data are usually presented as a $t$-integrated reduced cross section. For our analysis, we consider the diffractive reduced cross sections $\sigma_{r}^{D(4)}\left(\beta, Q^{2} ; x_{\mathbb{P}}, t\right)$ as presented in Eq. (1) with the contribution of $F_{L}^{D(4)}$.

It is well established now, from the factorization theorem for diffractive DIS in perturbative QCD [10-12], that the diffractive structure functions can be written as a convolution of hard scattering coefficient functions $C_{2 / L, i}$, with diffractive PDFs of flavor $i ; f_{i}^{D}\left(z, Q^{2} ; x_{\mathbb{P}}, t\right)$,

$$
F_{2 / L}^{D(4)}\left(\beta, Q^{2} ; x_{\mathbb{P}}, t\right)=\sum_{i} \int_{\beta}^{1} \frac{d z}{z} C_{2 / L, i}\left(\frac{\beta}{z}\right) f_{i}^{D}\left(z, Q^{2} ; x_{\mathbb{P}}, t\right) .
$$

In the above equation, the sum runs over all active partons including the quarks and gluons. The coefficient functions, $C_{2 / L, i}$, can be calculated perturbatively in QCD, whereas the diffractive parton densities, $f_{i}^{D}$, are nonperturbative quantities and should be determined through QCD global analyses of diffractive experimental datasets. It should be also noted that the diffractive PDFs, just like the usual parton densities in inclusive DIS, satisfy the wellknow Dokshitzer-Gribov-Lipatov-Altarelli-Parisi evolution equations [32-34]. Moreover, one can use the same coefficient functions, $C_{2 / L, i}$, as in inclusive DIS [35] for calculating diffractive structure functions of Eq. (2).

\section{B. Quark and gluon diffractive PDFs}

According to the Regge factorization hypothesis, the diffractive PDFs can be separated into two terms. One term involving the $x_{\mathbb{P}}$ and $t$ variables represents the Pomeron and Reggeon fluxes, and the other term, including the leptonic variables $\beta$ and $Q^{2}$, describes the hard scattering of the photon with the partonic structure of the Pomeron and Reggeon. Hence, considering the Regge factorization scheme, the diffractive PDFs can be formulated as

$$
\begin{aligned}
f_{i / p}^{D}\left(\beta, Q^{2} ; x_{\mathbb{P}}, t\right)= & f_{\mathbb{P} / p}\left(x_{\mathbb{P}}, t\right) f_{i / \mathbb{P}}\left(\beta, Q^{2}\right) \\
& +f_{\mathbb{R} / p}\left(x_{\mathbb{P}}, t\right) f_{i / \mathbb{R}}\left(\beta, Q^{2}\right),
\end{aligned}
$$

where the $f_{\mathbb{P} / p}$ and $f_{\mathbb{R} / p}$ are the Pomeron and Reggeon fluxes, respectively, and the $f_{i / \mathbb{P}}$ and $f_{i / \mathbb{R}}$ represent the parton densities of the Pomeron and Reggeon. It should be noted that one of the advantages of Regge factorization is the separation of the perturbative scale of the process, $Q^{2}$, from the $x_{\mathbb{P}}$ behavior that is genuinely nonperturbative. However, the normalization of the Pomeron flux is ambiguous, since the Pomeron is not a particle. In that way, the separation of the flux from the Pomeron density will be quite arbitrary.

As mentioned before, the dependence of the quark and gluon density functions of the Pomeron, $f_{i / \mathbb{P}}\left(\beta, Q^{2}\right)$, on the $Q^{2}$ scale can be obtained by the standard DokshitzerGribov-Lipatov-Altarelli-Parisi evolution equations, provided their dependence on $\beta$ is determined at an initial scale $Q_{0}^{2}$. Since the HERA diffractive DIS datasets could only constrain the sum of diffractive PDFs and, on the other hand, the available data are not sufficient enough to constrain all shape parameters of the separate flavors, diffractive PDFs are usually parametrized as simple functional forms at the initial scale in terms of quark $z f_{q}\left(z, Q_{0}^{2}\right)$ and gluon $z f_{g}\left(z, Q_{0}^{2}\right)$ distributions. The quark and antiquark distributions are assumed to be equal, $f_{u}=f_{d}=f_{s}=f_{\bar{u}}=f_{\bar{d}}=f_{\bar{s}}$.

It should be also noted that $z$ is the longitudinal momentum fraction of the struck parton with respect to the diffractive exchange that differs to $\beta$ when the higherorder processes are also included. In the present work, the Pomeron partonic densities are parametrized at the initial scale $Q_{0}^{2}=1.8 \mathrm{GeV}^{2}$ as follows: 


$$
\begin{aligned}
& z f_{q}\left(z, Q_{0}^{2}\right)=\alpha_{q} z^{\beta_{q}}(1-z)^{\gamma_{q}}\left(1+\eta_{q} \sqrt{z}\right), \\
& z f_{g}\left(z, Q_{0}^{2}\right)=\alpha_{g} z^{\beta_{g}}(1-z)^{\gamma_{g}}\left(1+\eta_{g} \sqrt{z}\right) .
\end{aligned}
$$

One should notice here that an extra factor $\exp [-0.001 /$ $(1-z)]$ is simply multiplied to the above parametrizations, in order to ensure that they go to zero for $z \rightarrow 1$. Considering the above parametrizations, the parameters $\gamma_{q}, \gamma_{g}, \eta_{q}$, and $\eta_{g}$ have the freedom in our analysis to extract from the QCD fit, so that can get negative or positive values. Such parametrizations have been extensively used in several analyses $[13,16,17]$, and their validity has been tested by HERA $e p$ experiments. For the case of Reggeon partonic densities in Eq. (3), we use the parametrization forms of the NLO Gluck-Reya-Vogt group, which have been obtained from a QCD analysis of the pion structure functions data; see Ref. [36] for details.

The dependence of the diffractive PDFs $f_{i}^{D}\left(\beta, Q^{2} ; x_{\mathbb{P}}, t\right)$ introduced in Eq. (3) to $x_{\mathbb{P}}$ is given by the flux factors of the Pomeron and Reggeon. In the present study, we use the same functional form as in Refs. [13,16,17],

$$
f_{\mathbb{P}, \mathbb{R}}\left(x_{\mathbb{P}}, t\right)=A_{\mathbb{P}, \mathbb{R}} \frac{e^{B_{\mathbb{P}, \mathbb{R}} t}}{x_{\mathbb{P}}^{2 \alpha_{\mathbb{R}, \mathbb{R}}(t)-1}},
$$

where $\alpha_{\mathbb{P}, \mathbb{R}}(t)$ is considered to be a linear function in terms of $t, \alpha_{\mathbb{P}, \mathbb{R}}(t)=\alpha_{\mathbb{P}, \mathbb{R}}(0)+\alpha_{\mathbb{P}, \mathbb{R}}^{\prime} t$. Hence, the Reggeon normalization factor, $A_{\mathbb{R}}$, and also the Pomeron and Reggeon intercepts, $\alpha_{\mathbb{P}}(0)$ and $\alpha_{\mathbb{R}}(0)$, are free parameters in our analysis and should be extracted from QCD fit to diffractive DIS datasets. Note that, according to Eq. (3) for diffractive PDFs, the value of Pomeron normalization parameter, $A_{\mathbb{P}}$, is absorbed in $\alpha_{q}$ and $\alpha_{g}$ parameters. The other parameters involving in Eq. (6) are considered to be constant with the values given in Ref. [13].

\section{Heavy quark contributions and numerical calculations}

It is worth noting that the evolution of diffractive PDFs is performed using the publicly available APFEL package [37]. Note also that, like for the case of ZEUS-2010-DPDFS [17] and GKG18 [13] analyses, the heavy quark contributions to the structure functions are considered using GM-VFNS [19-21]. Since this analysis is based on the APFEL package [37], we specifically use the FONLL-B GM-VFNS scheme [38] for our NLO QCD fits. Our GM-VFNS has a maximum of $N_{f}=5$ active quarks, and for the case of heavy quark masses, we fix the charm and bottom quark masses at $m_{c}=$ $1.40 \mathrm{GeV}$ and $m_{b}=4.75 \mathrm{GeV}$, respectively. As a last point, it should be mentioned that the value of the strong coupling constant at the $Z$-boson scale is considered to be $\alpha_{s}\left(M_{Z}\right)=0.1176$, consistent with the PDG average [39] and with very recent high-precision determinations [40-43]. In the present study, by performing a QCD analysis of the diffractive DIS data from HERA measurements in the presence of heay quark contributions, we obtain diffractive PDFs and their uncertainties at the NLO accuracy in QCD. To this aim, we use the CERN program library MINUIT [44] for performing the fit procedure and determining the unknown parameters.

\section{Higher twist effects}

As we discussed earlier in this article, we plan to show that the diffractive DIS data at low values of giga-electronvolts squared could provide the first evidence for the HT effects in diffractive DIS in the perturbative domain and hence could open the possibility for further theoretical and experimental investigations in such high-energy processes. In this section, our aim is to introduce the nonperturbative HT corrections to diffractive structure functions in the diffractive DIS experiment at HERA. In a wide kinematic region in terms of $x$ and $Q^{2}$, one can describe the structure functions of the DIS using leading twist (LT) corrections in QCD. However, for small values of $Q^{2}$ and large values of $x$, the structure functions in Eq. (1) should be corrected for some nonperturbative corrections. In general, in such a region, two types of corrections should be considered, which are the target mass corrections (TMCs) and the HT effects. In the case of TMCs, structure functions are corrected by modifying some quantities and adding new sentences, which can be found in Ref. [45] for full study in this area. For the HT case, it is customary to correct the leading twist structure function by adding a sentence that is inversely related to $Q^{2}$. More precisely, the higher twist effects are parametrized in the form of a phenomenological function. Then, one need to obtain the unknown parameters of this function by using the QCD fit to the experimental observables [46].

The phenomenological form for the HT effects in the corrected structure function is considered as

$$
F_{2}\left(x, Q^{2}\right)=F_{2}^{L T}\left(x, Q^{2}\right)\left(1+\frac{C_{H T}(x)}{Q^{2}}\right),
$$

where $F_{2}^{L T}$ represents the leading twist structure function, and the higher twist coefficient function is parametrized as follows:

$$
C_{H T}(x)=h_{0} x^{h_{1}}\left(1+h_{2} x\right) .
$$

The $h_{0}$ parameter represents the higher twist correction general scale, while $h_{1}$ controls the increase of the $C(x)$ coefficient at large $x$, and the parameter $h_{2}$ allows the probability of a higher twist at small values of momentum fraction $x$. In Eq. (8), $h_{i}\{i=0,1,2\}$ should be determined along with the fit parameters and then kept fixed.

To conclude this section, we should mentioned here that we follow a standard method to consider the higher twist effects in our QCD analysis of fully inclusive DIS structure 
functions. For a result of this study, we will show that such corrections are sizable at large values of the Bjorken variable $x$ and small region of $Q^{2}$. Detailed studies presented in Refs. [47-49] indicate that, in addition to this standard higher twist contribution, one could also consider the twist- 4 contribution, which dominates in the region of large $\beta$. This contribution comes from the diffractive production of the $q \bar{q}$ pair from the longitudinally polarized virtual photons $(L q \bar{q})$ in diffractive DIS. Formally, this contribution suppresses by a power of $1 / Q^{2}$ with respect to the leading twist- 2 contribution and is particularly important for the longitudinal diffractive structure function $F_{L}^{D}$. The results presented in Ref. [47] reveal that the diffractive gluon distribution obtained through a QCD fit with twist 4 has a stronger peak near large $\beta$. The longitudinal structure functions for the large value of $\beta \sim 1$ are also dominated by the twist- 4 contribution. Finally, we should note here that the scope of the present study is limited to the standard higher twist effects. The kinematical cuts that we applied on the datasets in our analysis are discussed in Sec. III B. For the $\beta$, we apply $\beta \leq 0.80$ over all datasets used in this study, and hence such a correction can be strongly reduced and safely ignored; thus, they are not considered.

\section{DIFRRACTIVE DIS DATASETS}

This section includes a comprehensive introduction to the diffractive DIS experimental datasets that we used in our diffractive PDFs analysis, discussing also the kinematical cuts and the treatment of experimental uncertainties.

\section{A. Experimental data}

In this analysis, we present all available inclusive diffractive DIS measurements on lepton-proton $(\ell p)$ scattering at HERA. In Fig. 2, we show the kinematical coverage in the $\beta$ and giga-electron-volts squared plane for the different experiments of diffractive DIS $e p$ scattering datasets used in this analysis. The dashed line represents the kinematic cuts applied on giga-electron-volts squared and $\beta$. The data points lying outside these lines shown in the figure are only excluded in the present QCD fits.

For our analysis, in particular, we use large rapidity gap (LRG) data from the H1 Collaboration [24,25] as well as the most recent data from H1/ZEUS combined inclusive diffractive cross section measurements [15].

In the following, we discuss each of these measurements in more detail. In addition to Fig. 2, a full list of all datasets has been presented in Table I as well. Note that for each dataset listed in this table we have provided the related published references; the kinematic intervals of $\beta, x_{\mathbb{P}}$, and $Q^{2}$ variables; and also the number of data points. As one can see, the included datasets cover a wide range of $\beta$ and $Q^{2}$, though they belong to small values of $x_{\mathbb{P}}$. It should be also noted that one needs to impose some kinematic cuts in order to avoid nonperturbative effects and also possible problems with the chosen theoretical framework. In this

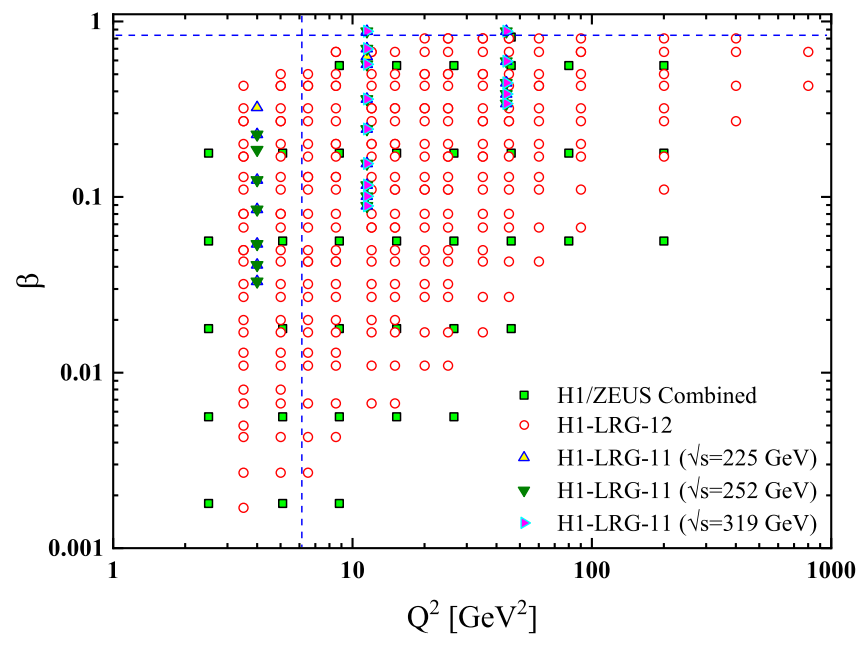

FIG. 2. Different experiments of diffractive DIS datasets in the $\beta$ and $Q^{2}$ plane. The dashed lines represent the kinematic cuts applied on $Q^{2}$ and $\beta$ in this analysis. The data points lying outside these lines shown in the figure are only excluded in the present QCD fits.

way, the total number of data points that are finally included in the analysis will be decreased after imposing related cuts. We discuss this issue in detail at the beginning of the next section, in which we investigate the appropriate value of the minimum cut on giga-electron-volts squared to consider the HT effects.

We have used three different datasets in this analysis. The latest H1/ZEUS combined dataset for the reduced diffractive cross sections, $\sigma_{r}^{D(3)}(e p \rightarrow e p X)$ [15], has been used. These calculations used samples of diffractive DIS $e p$ scattering data at a center-of-mass energy of $\sqrt{s}=$ $318 \mathrm{GeV}$ at the HERA collider. This precise measurement covers a range of photon virtualities $2.5 \mathrm{GeV}^{2}$ to $200 \mathrm{GeV}^{2}$ and $0.0018 \leq \beta \leq 0.816$. It should be noted that the most recent analyses from GKG18-DPDF [13], HK19-DPDF [18], and the analysis in Ref. [50] used the H1/ZEUS combined datasets in their analyses; however, those studies make no attempt to consider the beneficial effect arising from the inclusion of HT terms.

Another dataset is the measurement of inclusive diffractive DIS from H1-LRG-11, which was derived from the H1 detector in 2006 and 2007. These data correspond to three different center-of-mass energies, namely, $\sqrt{s}=225$, 252 , and $319 \mathrm{GeV}$ [24]. In these measurements, the reduced cross sections have been calculated in the range of photon virtualities $4 \mathrm{GeV}^{2} \leq Q^{2} \leq 44 \mathrm{GeV}^{2}$ for the center of mass $\sqrt{s}=225,252 \mathrm{GeV}$, and $11.5 \mathrm{GeV}^{2} \leq Q^{2} \leq 44 \mathrm{GeV}^{2}$ for the center of mass of $\sqrt{s}=319 \mathrm{GeV}$. The masses of the hadronic final state are in the range of $1.25 \leq M_{X} \leq 10.84$, and the proton vertex is considered to be $|t|<1 \mathrm{GeV}^{2}$. The diffractive variables are taken in the range of $5 \times 10^{-4} \leq x_{\mathbb{P}} \leq 3 \times 10^{-3}, 0.033 \leq \beta \leq 0.88$, and $0.089 \leq$ $\beta \leq 0.88$ for the center-of-mass energies of $\sqrt{s}=225,252$ and $=319 \mathrm{GeV}$, respectively. 
TABLE I. List of all diffractive DIS data points used in our global analysis. For each dataset, we have provided the corresponding publication reference, the kinematical coverage of $\beta, x_{\mathbb{P}}$, and $Q^{2}$. We have also displayed the number of data points before the baseline kinematical cuts. The details of kinematic cuts imposed on these data have been explained in detail in the text.

\begin{tabular}{|c|c|c|c|c|c|c|}
\hline Experiment & Observable & {$\left[\beta^{\min }, \beta^{\max }\right]$} & {$\left[x_{\mathbb{P}}^{\min }, x_{\mathbb{P}}^{\max }\right]$} & $\mathrm{GeV}^{2}$ & $\begin{array}{l}\text { No. of } \\
\text { points }\end{array}$ & Reference \\
\hline H1-LRG-11 $\sqrt{s}=225 \mathrm{GeV}$ & $\sigma_{r}^{D(3)}$ & {$[0.033-0.88]$} & {$\left[5 \times 10^{-4}-3 \times 10^{-3}\right]$} & $4-44$ & 22 & [24] \\
\hline H1-LRG-11 $\sqrt{s}=252 \mathrm{GeV}$ & $\sigma_{r}^{D(3)}$ & {$[0.033-0.88]$} & {$\left[5 \times 10^{-4}-3 \times 10^{-3}\right]$} & $4-44$ & 21 & [24] \\
\hline H1-LRG-11 $\sqrt{s}=319 \mathrm{GeV}$ & $\sigma_{r}^{D(3)}$ & {$[0.089-0.88]$} & {$\left[5 \times 10^{-4}-3 \times 10^{-3}\right]$} & $11.5-44$ & 14 & [24] \\
\hline H1-LRG-12 & $\sigma_{r}^{D(3)}$ & {$[0.0017-0.80]$} & {$\left[3 \times 10^{-4}-3 \times 10^{-2}\right]$} & $3.5-1600$ & 277 & [25] \\
\hline H1/ZEUS combined & $\sigma_{r}^{D(3)}$ & [0.0018-0.816] & {$\left[3 \times 10^{-4}-9 \times 10^{-2}\right]$} & $2.5-200$ & 192 & [15] \\
\hline Total data & & & & & 526 & \\
\hline
\end{tabular}

Finally, we use in this QCD fit the H1-LRG-12 data [25], in which the diffractive scattering $e p \rightarrow e X Y$ is corrected to the region $M_{Y}<1.6 \mathrm{GeV}$, and the momentum transfer of $|t|<1 \mathrm{GeV}^{2}$. They span the wide $x_{\mathbb{P}}$ range $0.0003 \leq x_{\mathbb{P}} \leq$ 0.03 and cover the ranges of $1.8 \times 10^{-3} \leq \beta \leq 0.88$ in $\beta$ and $3.5 \leq Q^{2} \leq 1600 \mathrm{GeV}^{2}$ in photon virtuality and $1.11 \leq$ $M_{X} \leq 48.99$.

\section{B. Kinematical cuts}

In this section, we briefly review the kinematical cuts applied on the diffractive DIS datasets analyzed in this study. In Fig. 2, we show the kinematical coverage in the $\left(\beta ; Q^{2}\right)$ plane of the diffractive DIS data included in our QCD fits. However, to minimize the contamination from low-scale nonperturbative corrections such as the target mass corrections and higher twist effects, one needs to impose some certain kinematical cuts on the $Q^{2}$ and the

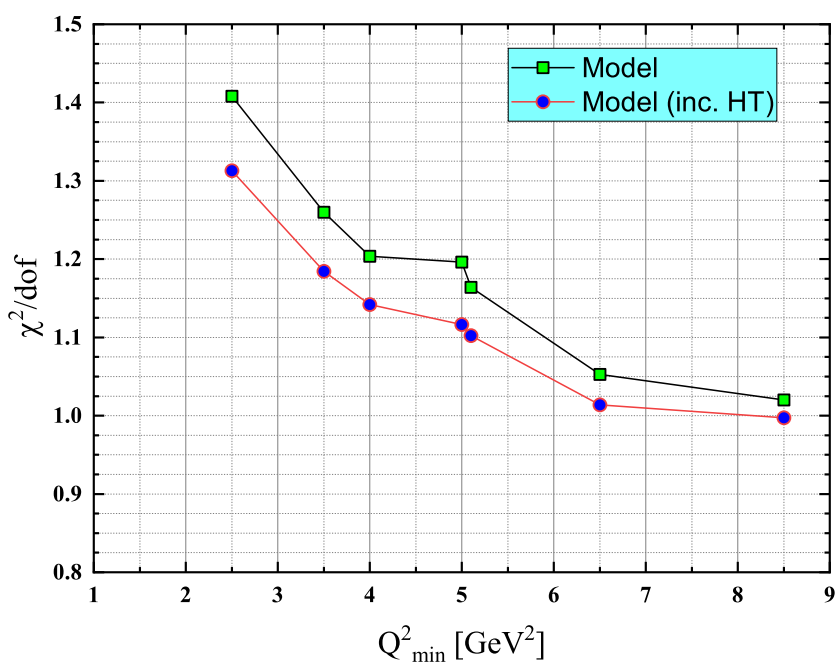

FIG. 3. The value of the total $\chi^{2} /$ d.o.f. vs the minimum $\mathrm{GeV}^{2}$ of data, $\mathrm{Q}_{\min }^{2}$, for all datasets entering in this study, which represents our specific choice of the kinematical cuts on the $Q_{\min }^{2}$. invariant final state mass $M_{X}$. The approach used in this investigation is similar to that used by other analyses in the literature (see, e.g., Refs. [13,16-18] for a clear review).

Like for the case of H1-2006 [16], ZEUS-2010 [17], GKG18 [13], and HK19-DPDF [18] QCD fits, we apply a cut on $M_{X}$, $\beta$, and $Q^{2}$. To determine our diffractive PDFs from the QCD fit, we apply $\beta \leq 0.80$ over all datasets used in this study. The data points with $M_{X}<2 \mathrm{GeV}$ are excluded from the fit.

However, the kinematical cut on $Q^{2}$ needs some more discussion. To this end, the dependence of the $\chi^{2}$ on the $Q^{2}$ cut applied to the analyzed datasets is investigated in our analysis, and the results are shown in Fig. 3. This figure shows the value of $\chi^{2}$ divided by the number of degrees of freedom, $\chi^{2} /$ d.o.f. vs the minimum giga-electron-volts squared of data included, $Q_{\min }^{2}$, for both of our QCD fits. Figure 3 interestingly shows that the $\chi^{2} /$ d.o.f. can be improved by the inclusions of the HT effects. In addition, as one can conclude from this figure, by increasing $Q^{2}$, no improvement can be seen for $\chi^{2} /$ d.o.f. As shown in this plot, a significant decrease in $\chi^{2} /$ d.o.f. can be seen at $Q_{\min }^{2} \leq 6.5 \mathrm{GeV}^{2}$, so the value of $\chi^{2} /$ d.o.f. is around the unity. Hence, we prefer to consider $Q_{\min }^{2}=6.5 \mathrm{GeV}^{2}$ as a best cut on $Q^{2}$. It is worth mentioning here that, in comparison to all other analyses in the literature that consider $8.5 \mathrm{GeV}^{2}$ in their QCD fits, our assumption enables us to use many more data points in our analysis. In fact, the total number of data points included in the analysis after imposing kinematical cuts is 499 , which shows that, according to Table I, only 27 data points are excluded.

\section{RESULTS OF THE DIFFRACTIVE PDFS ANALYSIS}

After introducing the phenomenological framework and experimental diffractive DIS datasets used in the present analysis, in this section, we aim to present the results obtained for diffractive PDFs with and without considering 
TABLE II. Parameters obtained with the different fits at the initial scale $Q_{0}^{2}=1.8 \mathrm{GeV}^{2}$ along with their experimental uncertainties. Parameters marked with the $*$ are fixed at their best-fit values.

\begin{tabular}{lcc}
\hline \hline Parameters & Model & Model (including HT) \\
\hline$\alpha_{q}$ & $0.376 \pm 0.029$ & $0.436 \pm 0.032$ \\
$\beta_{q}$ & $1.699 \pm 0.080$ & $1.541 \pm 0.072$ \\
$\gamma_{q}$ & $0.607 \pm 0.042$ & $0.723 \pm 0.042$ \\
$\eta_{q}$ & $0.0^{*}$ & $0.0^{*}$ \\
$\alpha_{g}$ & $2.166 \pm 0.468$ & $6.244 \pm 2.282$ \\
$\beta_{g}$ & $0.545 \pm 0.095$ & $0.831 \pm 0.137$ \\
$\gamma_{g}$ & $0.741 \pm 0.215$ & $2.400 \pm 0.509$ \\
$\eta_{g}$ & $0.0^{*}$ & $0.0^{*}$ \\
$\alpha_{\mathbb{P}}(0)$ & $1.091 \pm 0.003$ & $1.090 \pm 0.0031$ \\
$\alpha_{\mathbb{R}}(0)$ & $0.436 \pm 0.074$ & $0.437 \pm 0.080$ \\
$A_{\mathbb{R}}$ & $6.279 \pm 2.387$ & $6.279 \pm 2.582$ \\
$h_{0}$ & 0.0 & -25.798 \\
$h_{1}$ & 0.0 & 2.536 \\
$h_{2}$ & 0.0 & -1.381 \\
$\alpha_{s}\left(M_{Z}^{2}\right)$ & $0.1176^{*}$ & $0.1176^{*}$ \\
$m_{c}$ & $1.40^{*}$ & $1.40^{*}$ \\
$m_{b}$ & $4.750^{*}$ & $4.75^{*}$ \\
\hline \hline
\end{tabular}

the HT effects. To present more detailed discussions on our findings, we also compare the results with other available diffractive PDFs analyses from H1-2006 [16], ZEUS-2010 [17], and GKG18 [13]. Several comparisons of the NLO theoretical predictions and experimental data will be presented to check the quality of the fits in different kinematics. We mainly focus the regions of phase space where the HT has the largest impact on the quality of data/theory comparison.

We first start our discussions with the best-fit parameters obtained in this analysis. Table II contains the extracted fit parameters from our QCD analyses and "model (including HT)." Please note that the latter contains the results obtained by considering HT effects. As one can conclude from this table, almost all parameters are determined well, except the $\alpha_{g}$ for the case of HT analysis. The HT parameters, $h_{0}, h_{1}$, and $h_{2}$, are obtained in this fit along with the diffractive PDFs parameters also presented in the second part of the table. Parameters marked with the * have been fixed at their best-fit values. To give more flexibility to the parametrizations of Eq. (3), we let all parameters be free in the fit. Our investigations and the obtained results indicate that currently available diffractive DIS datasets would not be able to put further constraints on the $z$ dependence of the diffractive PDFs, suggesting more accurate experimental measurements need to be taken into account. In any case, we see from the first step of the minimization procedure that one cannot determine the $\eta$ parameter well enough. Hence, we prefer to fix this parameter to zero both for the quark and gluon densities.

To have better insight into which datasets led to better fit quality, let us now discuss our results in terms of the individual $\chi^{2}$ we obtained for each experiment and for both of our analyses. Table III contains the results of $\chi^{2}$ for both analyses of diffracive DIS data performed in the present work, with and without considering the HT terms. For each dataset, we have presented the related reference and also the value of $\chi^{2}$ divided by the number of analyzed data points $\left(\chi^{2} / N_{\mathrm{pts}}\right)$. Note that the last row of the table contains the values of $\chi^{2}$ per number of degrees of freedom, $\chi^{2} /$ d.o.f., for both analyses. As can be seen from this table, a reasonable improvement in the overall fit quality has been obtained after considering the HT contributions in the analysis. One can also conclude that the main improvements in the fit quality come mainly from the H1-LRG-12 [25] experimental data. For this dataset, we also see a much better $\chi^{2}$ after including the HT corrections. The improvement due to the HT terms also visible for the high-precision H1/ZEUS combined [15] experimental data.

After reviewing the individual $\chi^{2}$ for each dataset to judge the overall fit quality, we now discuss the extracted diffractive PDFs for both analyses. As we mentioned, one of the key ingredients in the strategy pursued in this analysis is the inclusion of HT terms. Hence, it should be interesting to see the significant change in the shape of the distributions after including the HT corrections.

In Fig. 4, our diffractive PDFs have been shown as a function of momentum fraction $z$ obtained at the input scale of $Q_{0}^{2}=1.8 \mathrm{GeV}^{2}$ for both of our analyses with and

TABLE III. The values of $\chi^{2} / N_{\text {pts }}$ for the datasets included in the analysis of diffractive PDFs without considering HT (second column) and by considering this term in the fit (third column).

\begin{tabular}{lcc}
\hline \hline & Without HT & With HT \\
\cline { 2 - 3 } Experiment & $\chi^{2} / N_{\mathrm{pts}}$ & $\chi^{2} / N_{\mathrm{pts}}$ \\
\hline H1-LRG-11 $\sqrt{s}=225 \mathrm{GeV}[24]$ & $15.595 / 20$ & $16.421 / 20$ \\
H1-LRG-11 $\sqrt{s}=252 \mathrm{GeV}[24]$ & $16.934 / 19$ & $17.834 / 19$ \\
H1-LRG-11 $\sqrt{s}=319 \mathrm{GeV}[24]$ & $7.220 / 12$ & $6.646 / 12$ \\
H1-LRG-12 [25] & $179.323 / 267$ & $165.654 / 267$ \\
H1/ZEUS combined [15] & $168.553 / 181$ & $163.606 / 181$ \\
$\chi^{2} /$ d.o.f. & $390.46 / 371=1.052$ & $376.079 / 371=1.013$ \\
\hline \hline
\end{tabular}



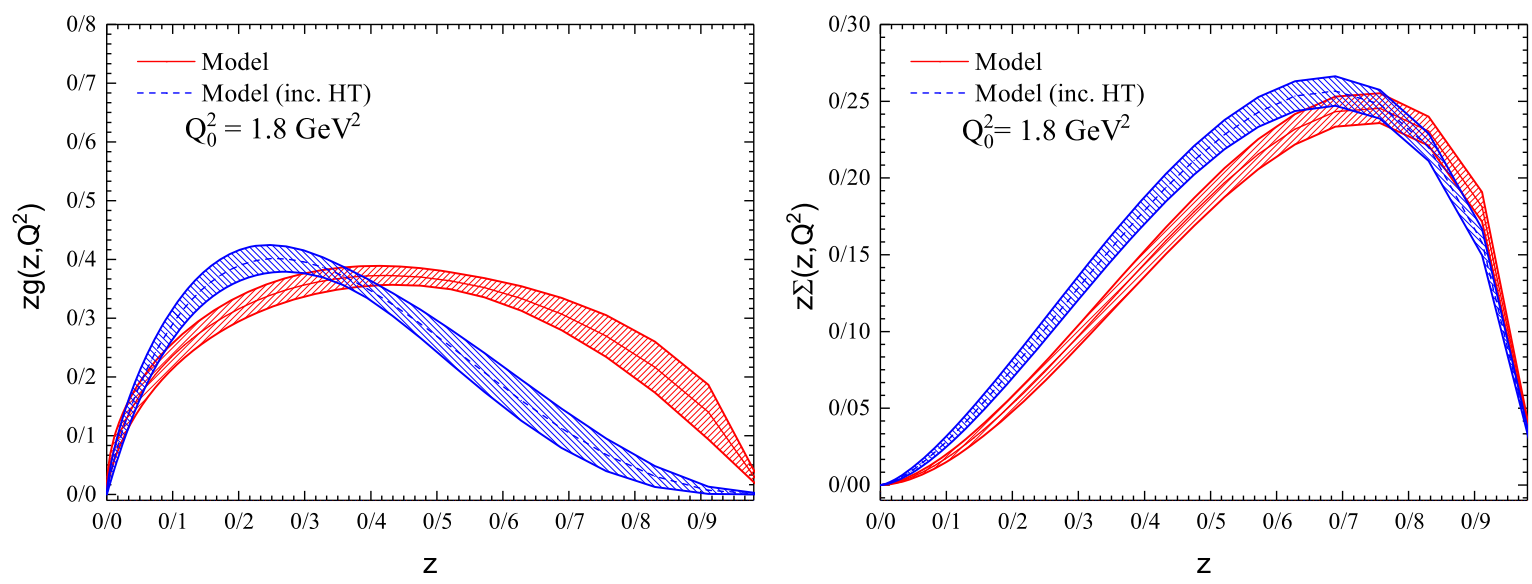

FIG. 4. The diffractive PDFs as a function of momentum fraction $z$ obtained at the input scale of $Q_{0}^{2}=1.8 \mathrm{GeV}^{2}$ for both of our analyses with and without the HT corrections. The error bands represent the uncertainty estimation coming from the experimental errors.

without the HT corrections. The error bands represent the uncertainty estimation coming from the experimental errors. It is also worth noticing here that for calculating the uncertainty bands for our diffractive PDFs and all theoretical predictions we use the standard "Hessian" error propagation [51-56] with $\Delta \chi^{2}=1$. For the case of predictions including HT effects, the related theoretical uncertainties are also included. As one can see in this plot, the inclusion of the HT term significantly changes the shape of the gluon density. One can see an enhancement for the small value of $z$ and a reduction for the larger value of $z$. For the quark density, one can also see an enhancement for the small value of $z$. It is also worth mentioning here that the kinematic region where the impact of the HT correction is most important is precisely the high region of $z$ at low $Q^{2}$, which mostly affects the shape of the gluon density. In terms of the comparison of error bands, one can see that the uncertainty bands for both of our analyses are almost similar in size, though a rather significant reduction is seen for the gluon density at large values of $z$ after including HT effects.

At this point, it is also enlightening to compare the diffractive PDFs obtained in this study with those of other available groups. These comparisons are shown in Fig. 5 with the results from H1-2006 fit B [16] and ZEUS-2010 fit SJ [17] for gluon and quark densities in three photon virtualities of $Q^{2}=6,20$, and $200 \mathrm{GeV}^{2}$. The $\mathrm{H} 1$ analysis has been obtained with the H1 LRG data, while ZEUS used for the first time the diffractive dijet production datasets. The error bands represent our uncertainty estimation using the Hessian method. The comparison of the results reveals the following conclusions. For the case of diffractive quark PDFs, one see the same patterns for all results. They show a pick around the medium value of $z, z \sim 0.6$. However, our results with the HT corrections are enhanced compared to those of other results for the region of $z<0.6$. For the gluon density, the comparisons are shown in the left panels of Fig. 5 for $Q^{2}=6,20$ and $200 \mathrm{GeV}^{2}$. The comparisons between the gluon densities show very different patterns. The different shapes for the gluon density reflect some reasons such as the different datasets used in these analyses. We have used the most recent $\mathrm{H} 1$ and ZEUS combined dataset, while H1-2006 used some older H1 LRG dataset. It is also worth mentioning here that the impact of the diffractive dijet production data on the gluon density function has been investigated by the ZEUS-2010 QCD analysis. The diffractive dijet production should also lead to the reduction of the gluon uncertainty. As one can expect from Fig. 5, for a higher value of photon virtuality $Q^{2}=200 \mathrm{GeV}^{2}$, all distributions follow the same trends.

It would be also interesting to compare the extracted quark and gluon densities with the most recent GKG18 [13] diffractive PDFs analysis. In Fig. 6, the comparison is shown for quark and gluon densities as a function of $z$ and for $\mathrm{Q}^{2}=6 \mathrm{GeV}^{2}$. Like for the case of Fig. 5, we see the same trend here, and hence the same conclusions hold for the comparisons between our results and the GKG18 analysis. GKG18 used the same datasets in its analysis with a different and bigger kinematical cut on $Q_{\min }^{2}$, and hence the number of data points that we use in the present analysis is more than in the GKG18 analysis. One of the main important results that can be concluded from this figure is the significant reduction in gluon density in the medium and large $z$ regions due to including HT effects in the analysis.

Although the main results of this analysis have been presented up to now, there are still other important discussions remaining in terms of the data/theory comparisons. To start our discussions, in the following, we present a detailed comparisons of our NLO theory predictions with almost all analyzed datasets in order to judge the fit quality and then to see the effect arising from the HT correction in some certain kinematical regions that are sensitive to this term. To this end, in Fig. 7, we compare our NLO 

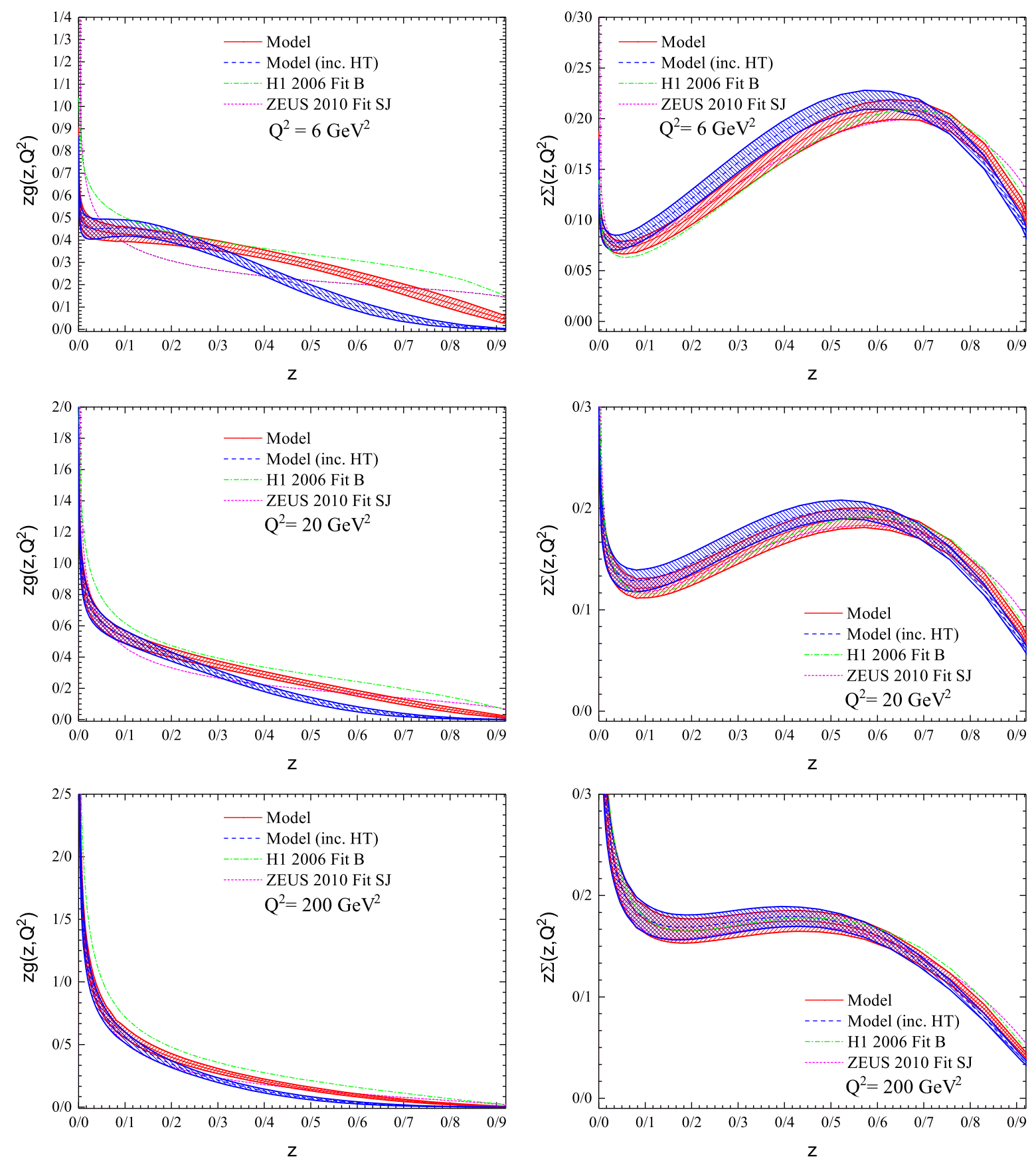

FIG. 5. The extracted diffractive PDFs for gluon and quark densities in three photon virtuality of $Q^{2}=6,20$ and $200 \mathrm{GeV}^{2}$ compared to the results of H1-2006 fit B [16] and ZEUS-2010 fit SJ [17].

theory prediction for the diffractive reduced cross sections $x_{\mathbb{P}} \sigma_{r}^{D(3)}\left(\beta, Q^{2} ; x_{\mathbb{P}}\right)$ with the recent $\mathrm{H} 1$ and ZEUS combined datasets [15]. In Figs. 8-12, the NLO theory predictions have been also shown as a function of $\beta$ for some selected values of $Q^{2}$ and for four representative bins of $x_{\mathbb{P}}=0.01$, $0.03,0.001$, and 0.003 . The dots show the central values of the experimental data points, and the data errors are defined by adding in quadrature the systematical and statistical uncertainties. These comparisons have been done for a wide range of $\beta, x_{\mathbb{P}}$, and $Q^{2}$ for both of our analyses with and without the presence of the HT correction. At the level of individual datasets and as expected by the $\chi^{2}$ values listed in Table III, these plots clearly show that the quality of the description between our NLO theory predictions and all the datasets analyzed in this study are quite acceptable.

With the agreements between data and theory established up to now, we are in a position to quantify in more detail the effect of HT corrections on the data/theory comparisons. However, from Table III, one expects a 

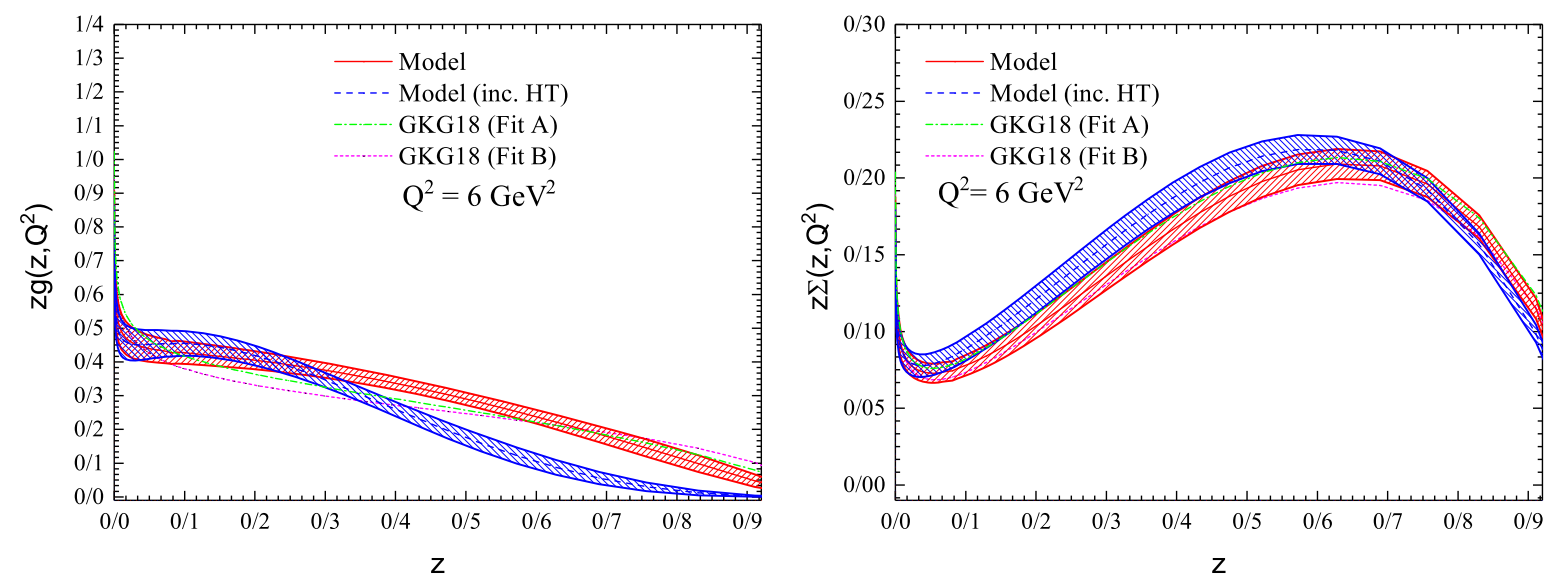

FIG. 6. The extracted diffractive PDFs for gluon and quark densities at $Q^{2}=6 \mathrm{GeV}^{2}$ compared to the most recent analysis by GKG18 [13].

better fit quality after the inclusion of HT terms. From the data vs theory comparisons, deviation among our results with and without the HT corrections for some certain region of $Q^{2}$ and $\beta$ can clearly be observed, for example, at $Q^{2}=6.5$ and $8.5 \mathrm{GeV}^{2}$ in Fig. 9 or at $Q^{2}=15 \mathrm{GeV}^{2}$ in Fig. 12. Let us now present comparisons between the

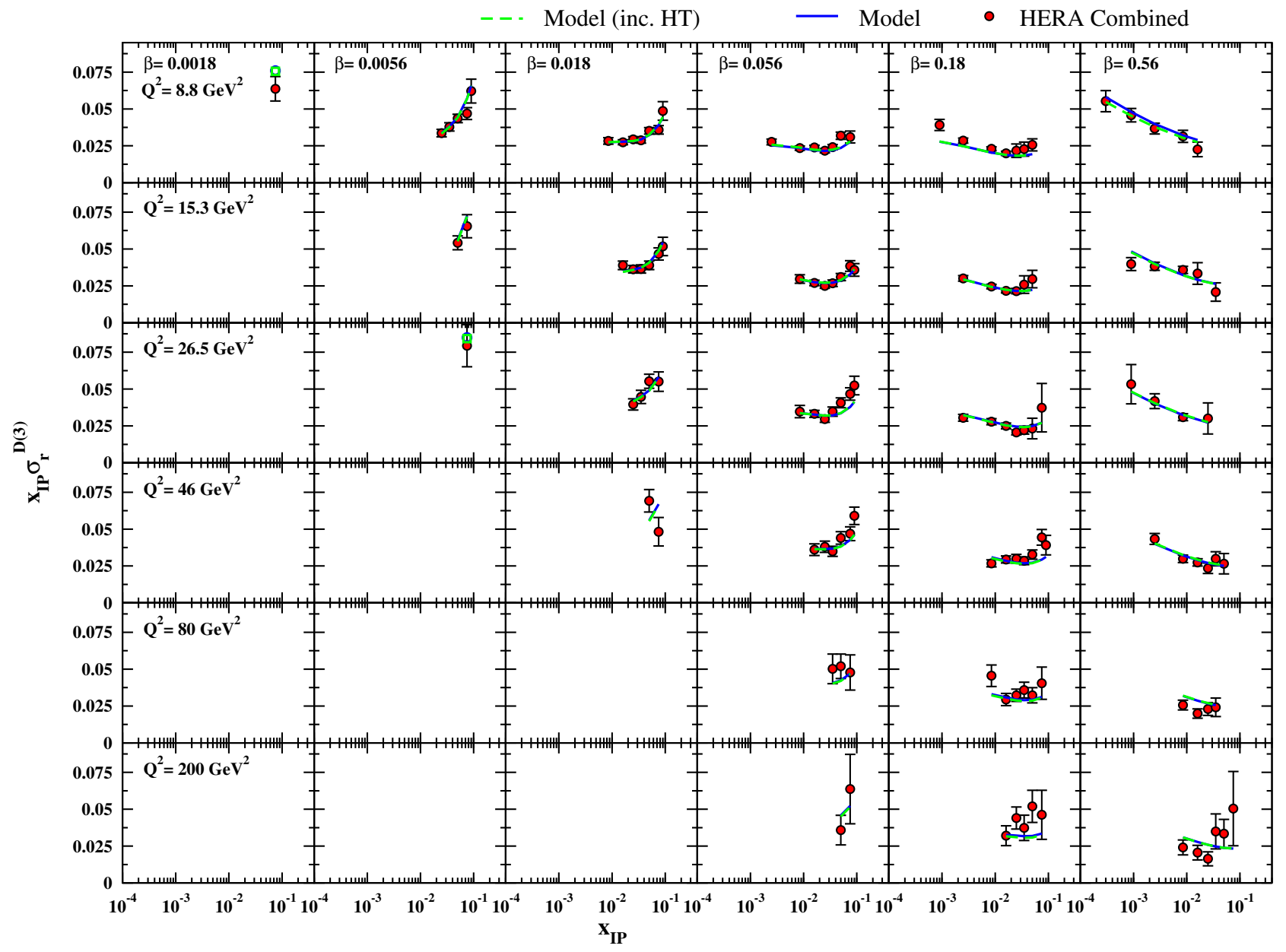

FIG. 7. Comparison between the experimental data on the diffractive reduced cross sections $x_{\mathbb{P}} \sigma_{r}^{D(3)}\left(\beta, Q^{2} ; x_{\mathbb{P}}\right)$ from the recent H1 and ZEUS combined dataset [15] and the corresponding NLO theoretical predictions from our NLO QCD fits without (solid curves) and with (dashed curves) considering HT effects. The dots show the central values of the experimental data points, and the data errors are defined by adding in quadrature the systematical and statistical uncertainties. 


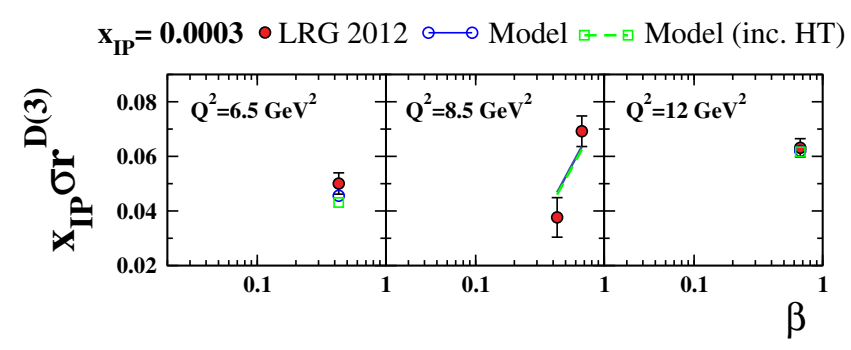

FIG. 8. Comparison between the experimental data on the diffractive reduced cross sections $x_{\mathbb{P}} \sigma_{r}^{D(3)}\left(\beta, Q^{2} ; x_{\mathbb{P}}\right)$ from the H1-LRG-12 datasets [25] and the corresponding NLO theoretical predictions from our NLO QCD fits without (solid curves) and with (dashed curves) considering HT effects. The comparisons have been done for the fixed value of $x_{\mathbb{P}}=0.0003$ and different photon virtuality $Q^{2}$. experimental data and the corresponding theoretical predictions. The results have been presented as a theory to data ratio. In Figs. 13, 14, and 15, we show these ratios in the presence of both of our results for some selected values of $Q^{2}=2.5,5.09$, and $8.8 \mathrm{GeV}^{2}$, respectively. The shaded bands correspond to the errors of experimental data points, which are obtained by adding the systematical and statistical uncertainties in quadrature. Here, the circle and triangle stand for the corresponding theoretical predictions for our analyses without and with the HT correction, respectively. Here, the lack of agreement between theory and data can be traced to the low- $Q^{2}$ region of the diffractive reduced cross section ratios. The disagreement in the low- $Q^{2}$ region is because we imposed $Q^{2}$ cuts on the datasets and the data below

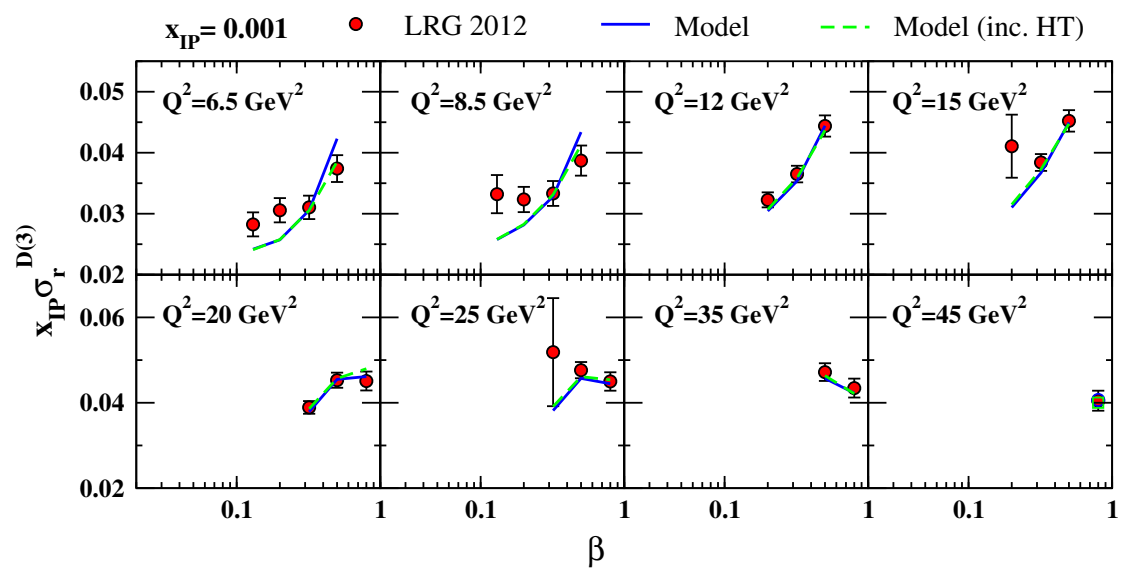

FIG. 9. Same as Fig. 8 but for the fixed value of $x_{\mathbb{P}}=0.001$.

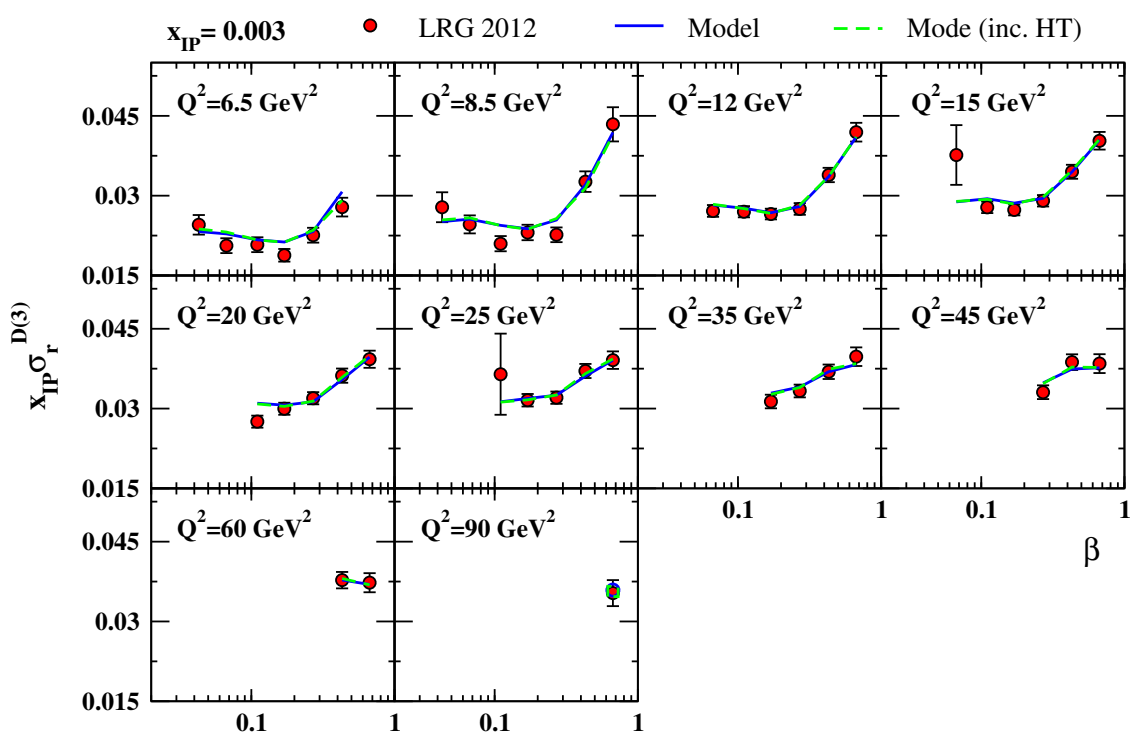

FIG. 10. Same as Fig. 8 but for the fixed value of $x_{\mathbb{P}}=0.003$. 


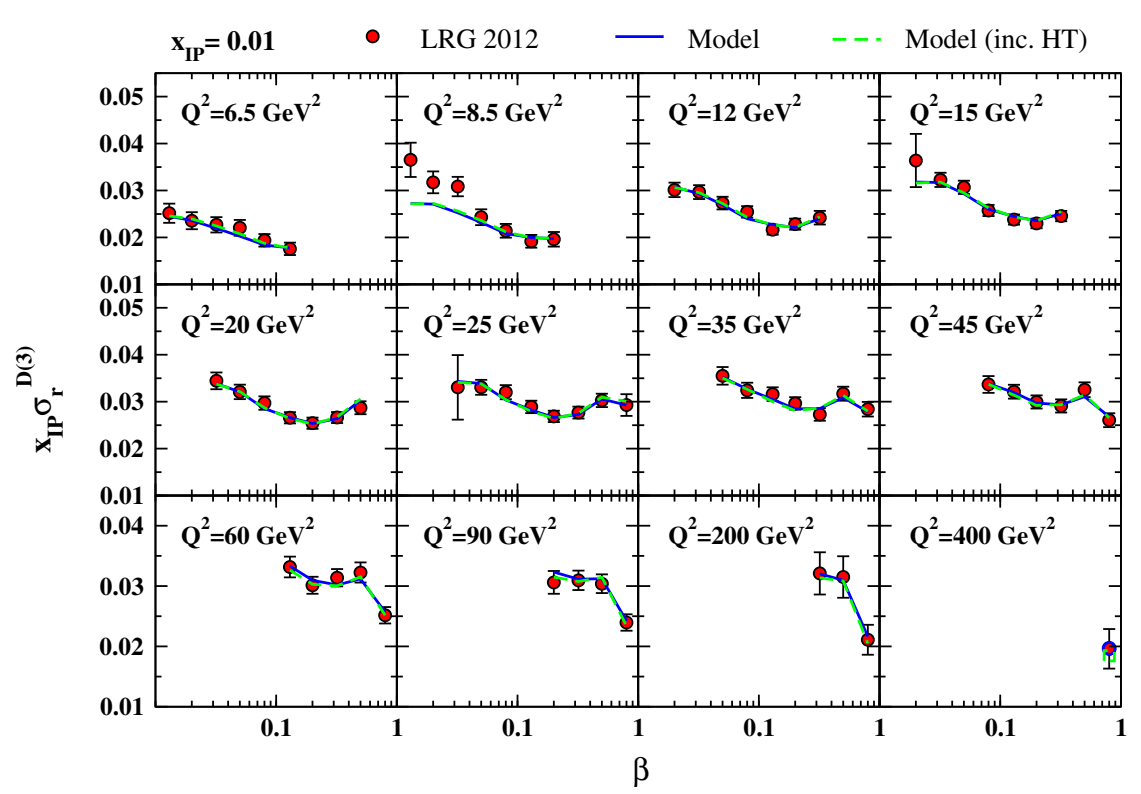

FIG. 11. Same as Fig. 8 but for the fixed value of $x_{\mathbb{P}}=0.01$.

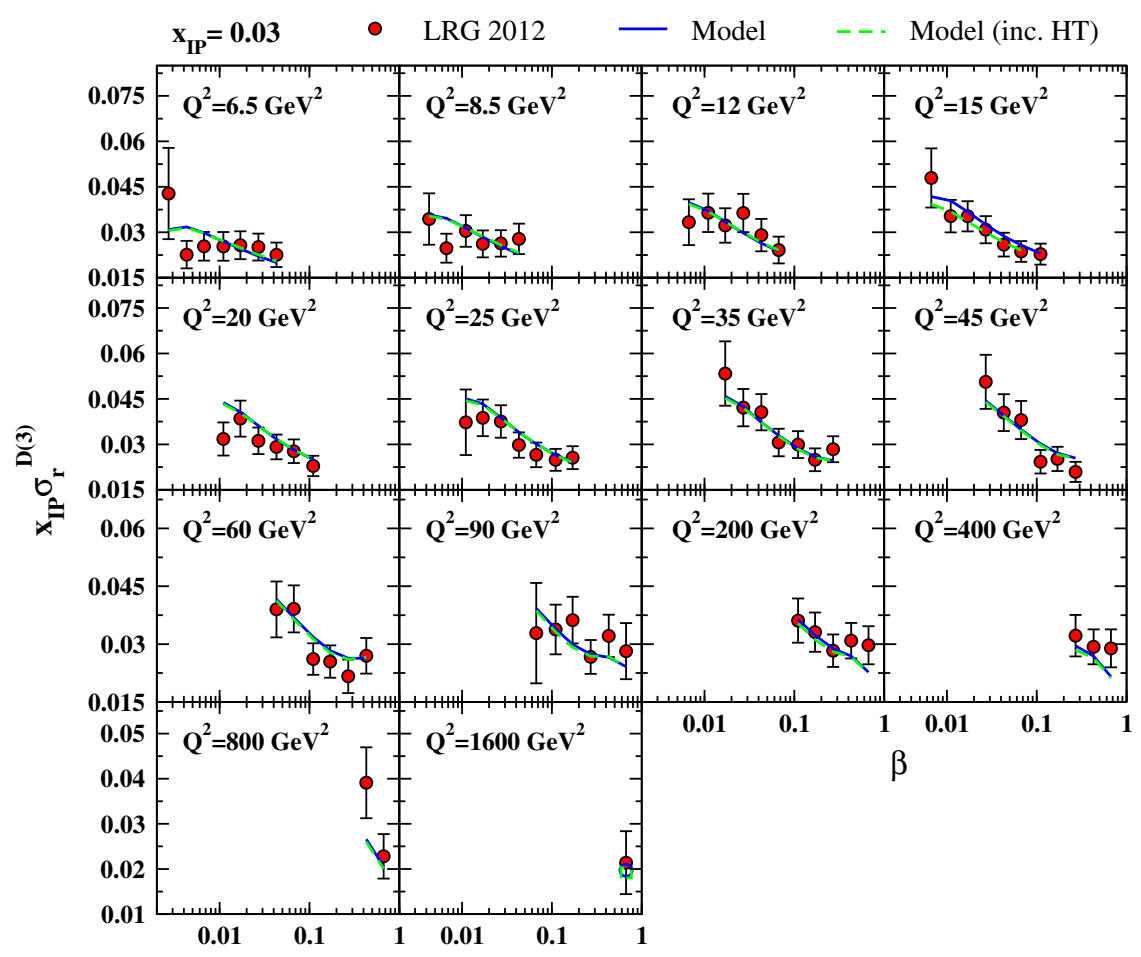

FIG. 12. Same as Fig. 8 but for the fixed value of $x_{\mathbb{P}}=0.03$.

$Q^{2}=Q_{\min }^{2} \leq 6.5$ have been excluded from the QCD fit. Overall, one can conclude from these figures that HT has more impact on theoretical predictions at lower $Q^{2}$ values, as expected, and becomes gradually ineffective as $Q^{2}$ increases. Another point that should be mentioned is that for smaller values of $Q^{2}$ HT has more impact on points with medium values of $\beta$, while as $Q^{2}$ increases, it affects points with larger values of $\beta$. For example, one can see from Figs. 13 and 14 that the greatest impact of HT on theoretical predictions related to $Q^{2}=2.5$ and $5.09 \mathrm{GeV}^{2}$ occurs at $\beta=1.78 \times 10^{-2}$ and $\beta=5.62 \times 10^{-2}$, so the differences between theory and data are decreased by including HT effects in the analysis. However, for data points with $Q^{2}=8.8 \mathrm{GeV}^{2}$, which are shown in 

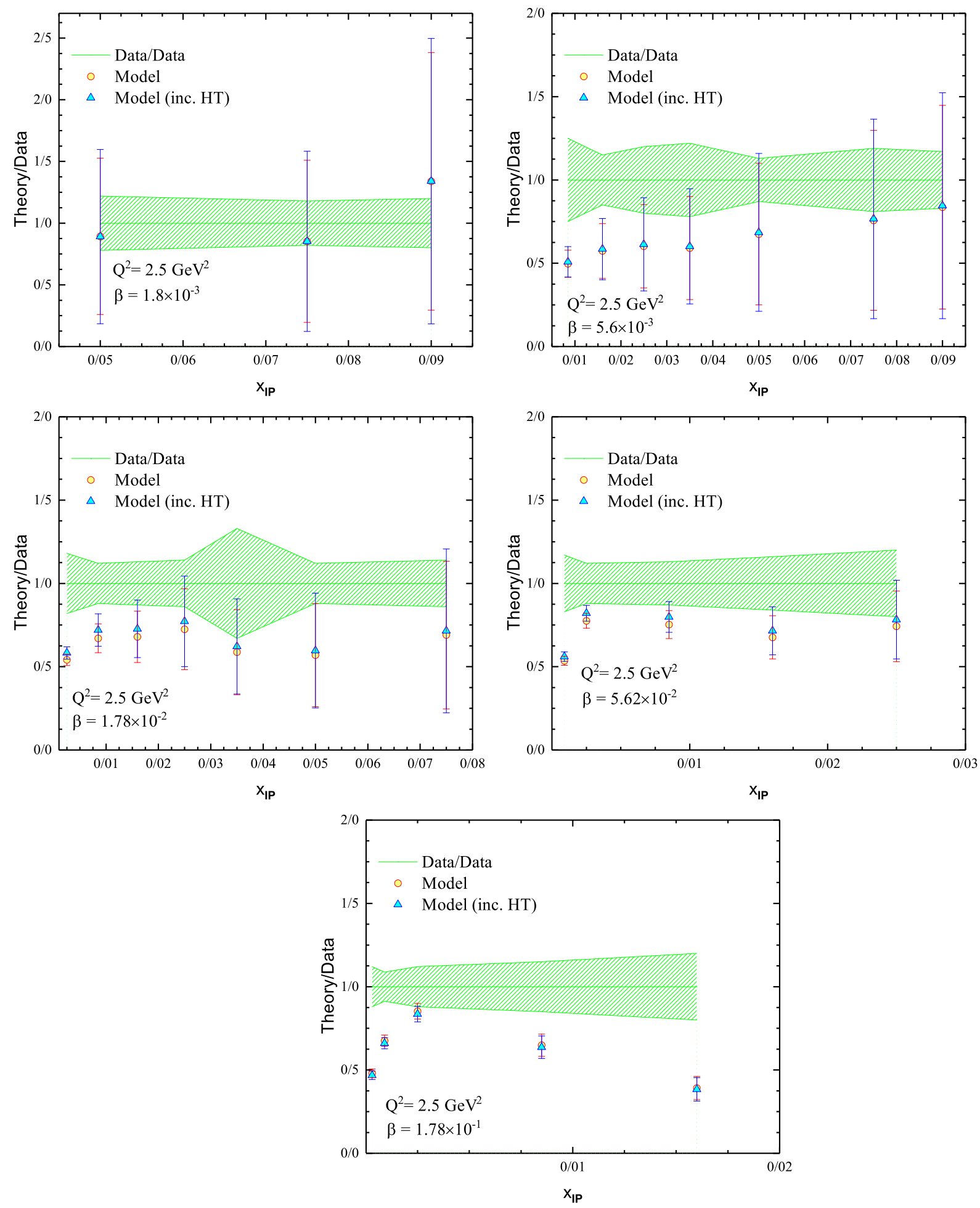

FIG. 13. Comparison between the experimental data from the $\mathrm{H} 1$ and ZEUS combined dataset [15] at $Q^{2}=2.5 \mathrm{GeV}^{2}$ and the corresponding theoretical predictions. The comparisons have been shown as theory to data ratios. Our results without HT effects are shown as a circle and the results including HT effects are presented as a triangle. The shaded bands correspond to the errors of experimental data points that are obtained by adding systematical and statistical uncertainties in quadrature.

Fig. 15, the greatest impact of HT occurs in the last panel, in which $\beta$ has larger values than before, namely, $\beta=5.62 \times 10^{-1}$.
For a short summary, we confirm the remarkable impact that HT correction would have on the diffractive PDFs, especially on those of the gluon density shape. Our study 

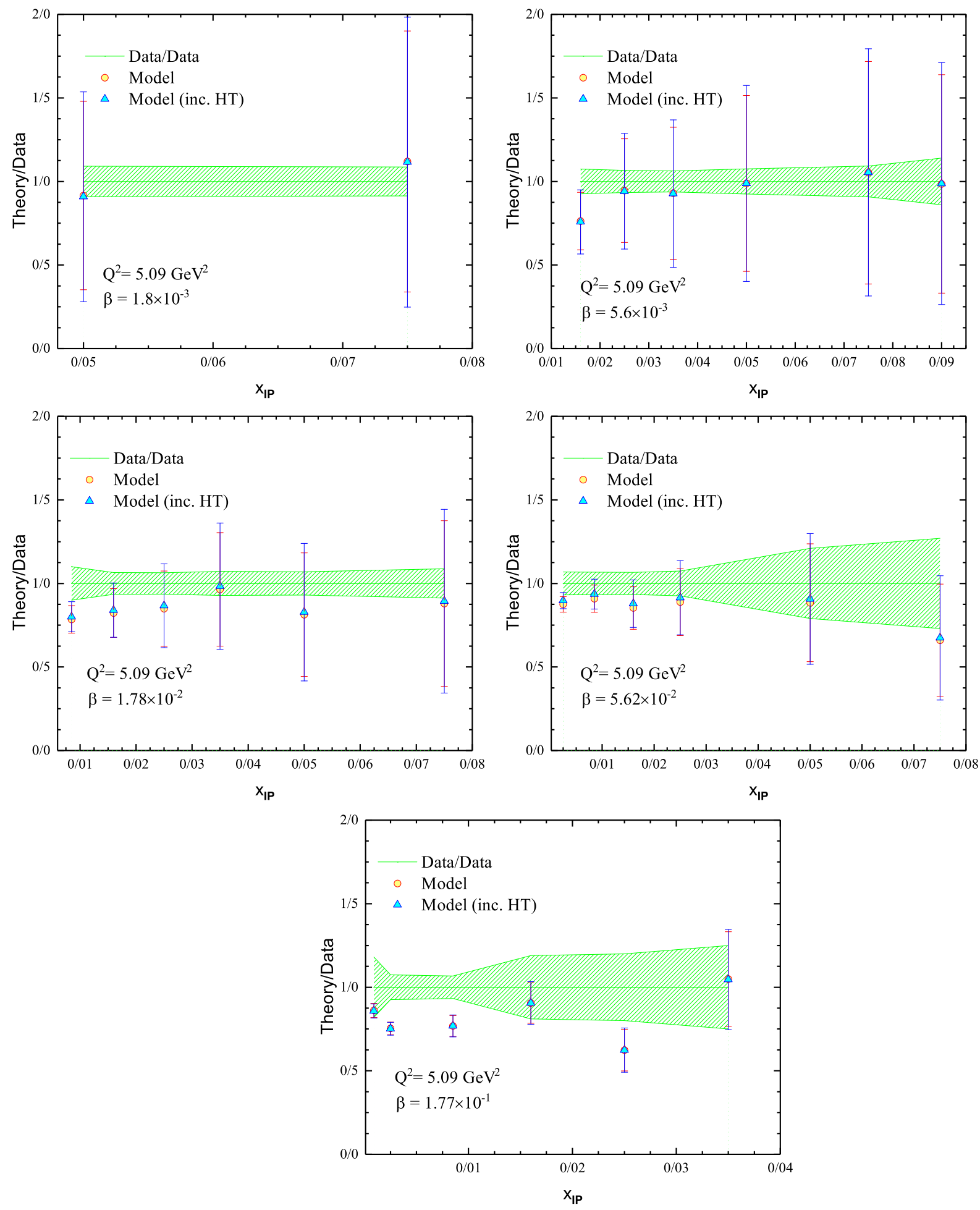

FIG. 14. Same as Fig. 13 but for $Q^{2}=5.09 \mathrm{GeV}^{2}$.

also suggests that the reductions in the uncertainties of extracted quark and gluon distributions are small. From the results presented in this section, we also find that the most significant effect on the total and individual $\chi^{2}$ will be achieved with the HT corrections and the much smaller kinematic cut on the $Q_{\min }^{2}$, which lead to the inclusion of more data points in the analysis. In the next section, we summarize and discuss our findings in this analysis and our outlook for future studies in which more stringent constraints can be found on diffractive PDFs. 

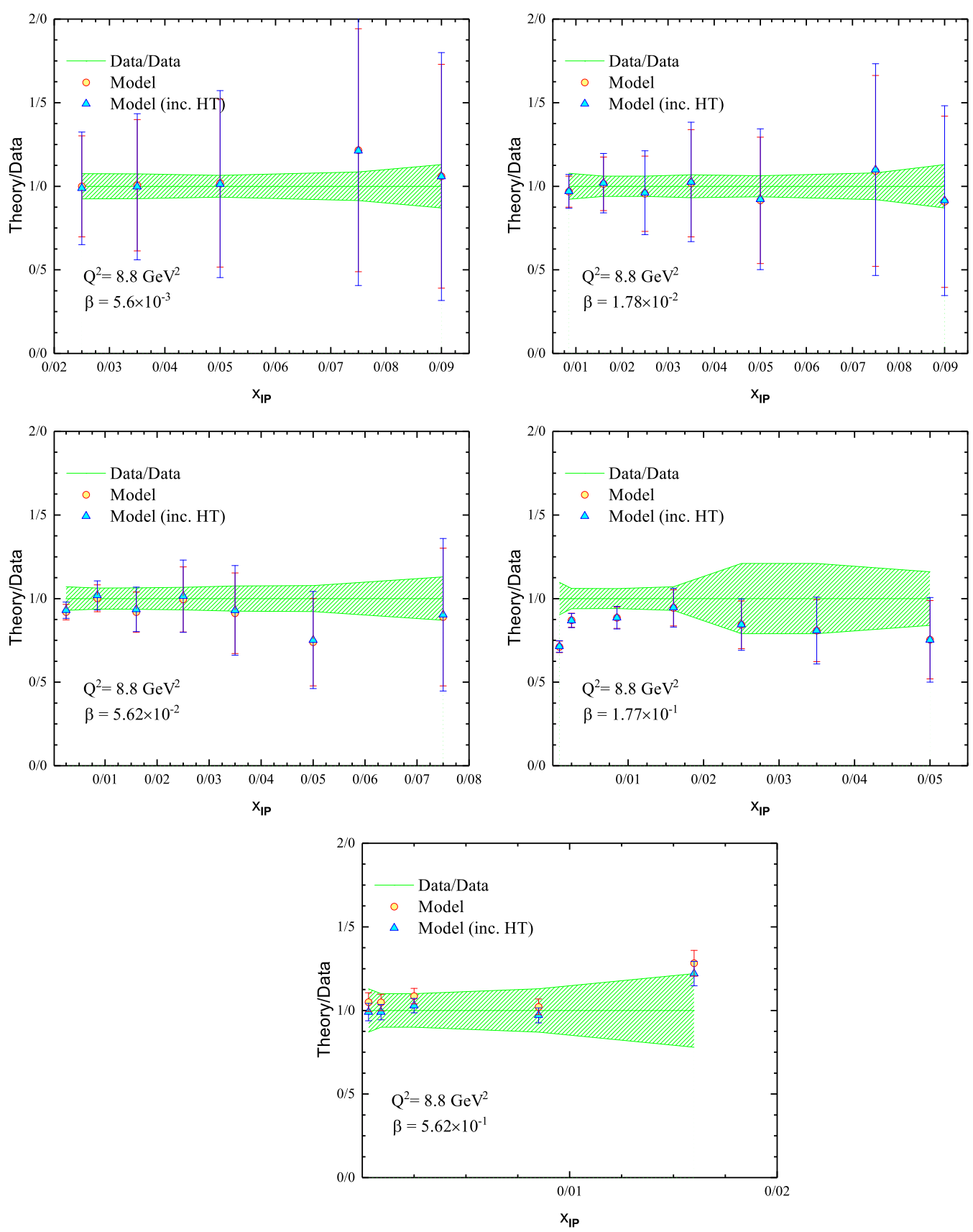

FIG. 15. Same as Fig. 13 but for $Q^{2}=8.8 \mathrm{GeV}^{2}$.

\section{CONCLUSIONS}

The diffractive process in deep-inelastic lepton-proton $(\ell p)$ scattering offers a remarkably versatile tool to probe the structure of the proton in terms of the quark and gluon density functions. It is well known that the QCD factorization theory allows one to model the diffractive DIS cross sections in terms of nonperturbative diffractive PDFs and hard scattering partonic cross sections in such a way that the precise measurements of the cross section can be used to extract the diffractive PDFs. The precision on such measurements could impose very stringent constraints on the quark and gluon distributions. To this end and in order to present a reliable and consistent determination of diffractive PDFs, we analyzed all available diffractive DIS datasets including the recent $\mathrm{H} 1$ /ZEUS combined diffractive cross section measurements and determined the diffractive PDFs along with their uncertainty bands evaluated with the Hessian approach.

Our analysis is also enriched with the HT contributions to the power corrections in diffractive DIS, which extend to small values of $Q^{2}$. It has been clearly demonstrated in this analysis that the HT terms in diffractive DIS are required for the kinematic coverage of the diffractive DIS 
processes at HERA. The most noticeable features that emerge from this study are the better fit quality and slightly significant reduction in the $\chi^{2}$ after considering the HT corrections.

Recent QCD studies clearly show that the key advantage of diffractive DIS data lies in the fact that they provide the possibility of determining the diffractive gluon and quark density functions. However, the combination of partonic flavors and more constraints on the gluon density also are challenging. Consequently, the unprecedented precision and kinematic coverage of the diffractive dijet measurements at HERA [57-59] and diffractive hadronic processes at the LHC certainly enhance our knowledge on diffractive PDFs and hence provide new insight into the inner structure of the nucleon in terms of its most basic constituents [7-9,60]. Another possible area of future research would be to investigate the effect arising from any available diffractive observable and possible theory developments in QCD analysis of diffractive PDFs. Considering any new improvements, future studies on the current topic are therefore recommended.

\section{ACKNOWLEDGMENTS}

H. M. and A. M. are grateful to Semnan University for financial support for this project. H. K. and M. G. thank School of Particles and Accelerators, Institute for Research in Fundamental Sciences for financial support for this research. H. K. is also thankful for the University of Science and Technology of Mazandaran for financial support provided for this project and the CERN theory department for its hospitality and support during the preparation of this paper.
[1] J. Gao, L. Harland-Lang, and J. Rojo, The structure of the proton in the LHC precision era, Phys. Rep. 742, 1 (2018).

[2] R. A. Khalek et al., A first determination of parton distributions with theoretical uncertainties, arXiv:1905.04311.

[3] R. A. Khalek, S. Bailey, J. Gao, L. Harland-Lang, and J. Rojo, Towards ultimate parton distributions at the highluminosity LHC, Eur. Phys. J. C 78, 962 (2018).

[4] L. Rottoli, Precision QCD at the LHC: From the structure of the proton to all-order resummations, arXiv:1810.08257.

[5] H. W. Lin et al., Parton distributions and lattice QCD calculations: A community white paper, Prog. Part. Nucl. Phys. 100, 107 (2018).

[6] B. Schmookler et al. (CLAS Collaboration), Modified structure of protons and neutrons in correlated pairs, Nature (London) 566, 354 (2019).

[7] C. O. Rasmussen and T. Sjöstrand, Models for total, elastic and diffractive cross sections, Eur. Phys. J. C 78, 461 (2018).

[8] I. Helenius and C. O. Rasmussen, Hard diffraction in photoproduction with Pythia 8, Eur. Phys. J. C 79, 413 (2019).

[9] D. Britzger, J. Currie, T. Gehrmann, A. Huss, J. Niehues, and R. Žlebík, Dijet production in diffractive deep-inelastic scattering in next-to-next-to-leading order QCD, Eur. Phys. J. C 78, 538 (2018).

[10] J. C. Collins, L. Frankfurt, and M. Strikman, Factorization for hard exclusive electroproduction of mesons in QCD, Phys. Rev. D 56, 2982 (1997).

[11] J. C. Collins, Factorization in hard diffraction, J. Phys. G 28, 1069 (2002).

[12] J. C. Collins, Proof of factorization for diffractive hard scattering, Phys. Rev. D 57, 3051 (1998); Erratum, Phys. Rev. D 61, 019902(E) (1999).

[13] M. Goharipour, H. Khanpour, and V. Guzey, First global next-to-leading order determination of diffractive parton distribution functions and their uncertainties within the Fitter framework, Eur. Phys. J. C 78, 309 (2018).

[14] S. Alekhin et al., HERAFitter, Eur. Phys. J. C 75, 304 (2015).

[15] F. D. Aaron et al. (H1 and ZEUS Collaborations), Combined inclusive diffractive cross sections measured with forward proton spectrometers in deep inelastic ep scattering at HERA, Eur. Phys. J. C 72, 2175 (2012).

[16] A. Aktas et al. (H1 Collaboration), Measurement and QCD analysis of the diffractive deep-inelastic scattering cross-section at HERA, Eur. Phys. J. C 48, 715 (2006).

[17] S. Chekanov et al. (ZEUS Collaboration), A QCD analysis of ZEUS diffractive data, Nucl. Phys. B831, 1 (2010).

[18] H. Khanpour, Phenomenology of diffractive DIS in the framework of fracture functions and determination of diffractive parton distribution functions, Phys. Rev. D 99, 054007 (2019).

[19] L. A. Harland-Lang, A. D. Martin, P. Motylinski, and R. S. Thorne, Parton distributions in the LHC era: MMHT 2014 PDFs, Eur. Phys. J. C 75, 204 (2015).

[20] R. S. Thorne and R. G. Roberts, An ordered analysis of heavy flavor production in deep inelastic scattering, Phys. Rev. D 57, 6871 (1998).

[21] R. S. Thorne, A variable-flavor number scheme for NNLO, Phys. Rev. D 73, 054019 (2006).

[22] C. Royon, L. Schoeffel, S. Sapeta, R. B. Peschanski, and E. Sauvan, A global analysis of inclusive diffractive cross sections at HERA, Nucl. Phys. B781, 1 (2007).

[23] S. Chekanov et al. (ZEUS Collaboration), Deep inelastic scattering with leading protons or large rapidity gaps at HERA, Nucl. Phys. B816, 1 (2009).

[24] F. D. Aaron et al. (H1 Collaboration), Measurement of the diffractive longitudinal structure function $F_{L}^{D}$ at HERA, Eur. Phys. J. C 71, 1836 (2011). 
[25] F. D. Aaron et al. (H1 Collaboration), Inclusive measurement of diffractive deep-inelastic scattering at HERA, Eur. Phys. J. C 72, 2074 (2012).

[26] A. Aktas et al. (H1 Collaboration), Diffractive open charm production in deep-inelastic scattering and photoproduction at HERA, Eur. Phys. J. C 50, 1 (2007).

[27] F. D. Aaron et al. (H1 Collaboration), Measurement of beauty and charm photoproduction using semi-muonic decays in dijet events at HERA, Eur. Phys. J. C 72, 2047 (2012).

[28] A. Aktas et al. (H1 Collaboration), Tests of QCD factorisation in the diffractive production of dijets in deep-inelastic scattering and photoproduction at HERA, Eur. Phys. J. C 51, 549 (2007).

[29] S. Chekanov et al. (ZEUS Collaboration), Deep inelastic inclusive and diffractive scattering at $Q^{2}$ values from 25 to $320 \mathrm{GeV}^{2}$ with the ZEUS forward plug calorimeter, Nucl. Phys. B800, 1 (2008).

[30] S. Chekanov et al. (ZEUS Collaboration), Study of deep inelastic inclusive and diffractive scattering with the ZEUS forward plug calorimeter, Nucl. Phys. B713, 3 (2005).

[31] S. Chekanov et al. (ZEUS Collaboration), Dissociation of virtual photons in events with a leading proton at HERA, Eur. Phys. J. C 38, 43 (2004).

[32] A. Berera and D. E. Soper, Behavior of diffractive parton distribution functions, Phys. Rev. D 53, 6162 (1996).

[33] A. D. Martin, M. G. Ryskin, and G. Watt, Diffractive parton distributions from H1 data, Phys. Lett. B 644, 131 (2007).

[34] Z. Kunszt and W. J. Stirling, Hard diffractive scattering: Partons and QCD, arXiv:hep-ph/9609245.

[35] J. A. M. Vermaseren, A. Vogt, and S. Moch, The Thirdorder QCD corrections to deep-inelastic scattering by photon exchange, Nucl. Phys. B724, 3 (2005).

[36] M. Gluck, E. Reya, and A. Vogt, Parton distributions for high-energy collisions, Z. Phys. C 53, 127 (1992),

[37] V. Bertone, S. Carrazza, and J. Rojo, APFEL: A PDF evolution library with QED corrections, Comput. Phys. Commun. 185, 1647 (2014).

[38] S. Forte, E. Laenen, P. Nason, and J. Rojo, Heavy quarks in deep-inelastic scattering, Nucl. Phys. B834, 116 (2010).

[39] M. Tanabashi et al. (Particle Data Group), Review of particle physics, Phys. Rev. D 98, 030001 (2018).

[40] M. Bruno, M. Dalla Brida, P. Fritzsch, T. Korzec, A. Ramos, S. Schaefer, H. Simma, S. Sint, and R. Sommer (ALPHA Collaboration), QCD Coupling from a Nonperturbative Determination of the Three-Flavor $\Lambda$ Parameter, Phys. Rev. Lett. 119, 102001 (2017).

[41] A. Verbytskyi et al., High precision determination of $\alpha_{s}$ from a global fit of jet rates, arXiv:1902.08158.

[42] R. D. Ball, S. Carrazza, L. Del Debbio, S. Forte, Z. Kassabov, J. Rojo, E. Slade, and M. Ubiali (NNPDF Collaboration), Precision determination of the strong coupling constant within a global PDF analysis, Eur. Phys. J. C 78, 408 (2018).
[43] S. Zafeiropoulos, P. Boucaud, F. De Soto, J. RodríguezQuintero, and J. Segovia, Strong Running coupling from the Gauge Sector of Domain Wall Lattice QCD with Physical Quark Masses, Phys. Rev. Lett. 122, 162002 (2019).

[44] F. James and M. Roos, Minuit: A system for function minimization and analysis of the parameter errors and correlations, Comput. Phys. Commun. 10, 343 (1975).

[45] I. Schienbein et al., A review of target mass corrections, J. Phys. G 35, 053101 (2008).

[46] A. Accardi, L. T. Brady, W. Melnitchouk, J. F. Owens, and N. Sato, Constraints on large- $x$ parton distributions from new weak boson production and deep-inelastic scattering data, Phys. Rev. D 93, 114017 (2016).

[47] K. J. Golec-Biernat and A. Luszczak, Diffractive parton distributions from the analysis with higher twist, Phys. Rev. D 76, 114014 (2007).

[48] K. Golec-Biernat, Diffractive parton distributions from the analysis with higher twist, AIP Conf. Proc. 1105, 205 (2009).

[49] K. J. Golec-Biernat and M. Wusthoff, Diffractive parton distributions from the saturation model, Eur. Phys. J. C 20, 313 (2001).

[50] F. A. Ceccopieri, Single-diffractive Drell-Yan pair production at the LHC, Eur. Phys. J. C 77, 56 (2017).

[51] A. D. Martin, R. G. Roberts, W. J. Stirling, and R. S. Thorne, Uncertainties of predictions from parton distributions. 2. Theoretical errors, Eur. Phys. J. C 35, 325 (2004).

[52] J. Pumplin, D. Stump, R. Brock, D. Casey, J. Huston, J. Kalk, H. L. Lai, and W. K. Tung, Uncertainties of predictions from parton distribution functions. 2. The Hessian method, Phys. Rev. D 65, 014013 (2001).

[53] A. D. Martin, W. J. Stirling, R. S. Thorne, and G. Watt, Parton distributions for the LHC, Eur. Phys. J. C 63, 189 (2009).

[54] D. de Florian, R. Sassot, P. Zurita, and M. Stratmann, Global analysis of nuclear parton distributions, Phys. Rev. D 85, 074028 (2012).

[55] C. Schmidt, J. Pumplin, C. P. Yuan, and P. Yuan, Updating and optimizing error parton distribution function sets in the Hessian approach, Phys. Rev. D 98, 094005 (2018).

[56] K. J. Eskola, H. Paukkunen, and C. A. Salgado, EPS09: A new generation of NLO and LO nuclear parton distribution functions, J. High Energy Phys. 04 (2009) 065.

[57] S. Chekanov et al. (ZEUS Collaboration), Measurement of dijet photoproduction for events with a leading neutron at HERA, Nucl. Phys. B827, 1 (2010).

[58] V. Andreev et al. (H1 Collaboration), Diffractive dijet production with a leading proton in $e p$ collisions at HERA, J. High Energy Phys. 05 (2015) 056.

[59] V. Andreev et al. (H1 Collaboration), Measurement of dijet production in diffractive deep-inelastic ep scattering at HERA, J. High Energy Phys. 03 (2015) 092.

[60] V. P. Gonçalves, R. P. da Silva, and P. V. R. G. Silva, Diffractive excitation in $p p$ and $p A$ collisions at high energies, Phys. Rev. D 100, 014019 (2019). 